\title{
Abuso de la operación cesárea y el principio de beneficencia
}

\section{The abuse of cesarean and the principle of beneficence}

\section{O abuso da cirurgia cesariana e do principio da beneficência}

\author{
Fecha de recepción: 13 de junio de 2015 \\ Fecha de evaluación: 17 de agosto de 2015 \\ Fecha de aceptación: 26 de octubre de 2015 \\ Disponible en línea: 15 de diciembre de 2015 \\ José Carlos Vázquez Parra* \\ DOI: http://dx.doi.org/10.18359/rlbi.1441
}

Cómo citar:

Vázquez Parra, J. C. (2016). Abuso de la operación cesárea y el principio de beneficencia. Revista Latinoamericana de Bioética, 16(1), 60-71. DOI: http://dx.doi.org/10.18359/rlbi.1441.

Doctor en Estudios Humanísticos con especialidad en ética, maestro en Educación y Licenciado en Derecho y en Psicología. Profesor de cátedra, Departamento de Formación Humanística y Ciudadana del Tecnológico de Monterrey, Zapopan, Jalisco, México. jcvazquezp@itesm.mx. 


\section{Resumen}

Hablar de bioética en la época contemporánea nos obliga a hacer referencia a algunos temas que día a día generan controversias en el área de la medicina y la salud. Sin lugar a duda uno de estos temas es el que tiene relación con el uso excesivo y en ocasiones innecesario de la operación cesárea, la cual, aunque debería ser una cirugía reservada únicamente a casos en los que el parto por vía vaginal sea riesgoso, en la actualidad se constituye como una alternativa que ya no toma de manera exclusiva y razonada el médico, sino que incluso es una opción que el mismo paciente elige. El presente artículo tiene por objetivo hacer un planteamiento ético sobre la práctica de la operación cesárea, considerando que aunque es obligación del médico respetar la autonomía de sus pacientes, también es su deber el orientarlos sobre los beneficios y riesgos de cualquiera de sus decisiones. Los principios de bioética del profesionista de la salud nos permiten hacer una valoración del ejercicio médico, poniendo especial atención en la decisión de elegir de manera racional una operación cesárea sobre el parto por vía vaginal.

Palabras clave: beneficencia, cesárea, parto, médico, autonomía.

\section{Abstract}

Talk of bioethics in modern times forces us to refer to some issues daily generate controversies in the field of medicine and health. Without a doubt, one of these topics is the unnecessary, and sometimes, excessive use of cesarean section. This surgery should be reserved only for cases where vaginal childbirth is risky, and it is now seen as an alternative that takes no longer exclusively medical and reasoned, but even is an option that the patient chooses. This article aims to make an ethical approach to the practice of cesarean section, considering that while it is the duty of the doctors to respect the autonomy of their patients, it is also their duty to guide them through the benefits and risks of any their decisions. The principles of bioethics of the professional health let us make an assessment of medical practice, paying special attention to the decision to choose rationally, a cesarean section instead a vaginal delivery.

Keywords: Beneficence, cesarean, childbirth, doctor, autonomy.

\section{Resumo}

Falar da bioética na época contemporânea nos força para nós referirmos a algumas questões que no dia a dia geram polêmicas na área da medicina e da saúde. Sem dúvida alguma, um desses temas é aquele que tem relação ao uso excessivo e em muitas ocasiões desnecessário da cirurgia da cesariana, a qual, embora a intervenção cirúrgica tivesse que ser reservada apenas para os casos em que o parto por via vaginal seja arriscado, na atualidade é constituída como uma alternativa que já não é tomada de maneira exclusiva e fundamentada o médico, mas ainda é uma opção que a mesmo paciente pode escolher. O presente artigo tem como objetivo fazer uma abordagem ética sobre a prática da intervenção cirúrgica da cesariana, considerando que, embora seja dever do médico respeitar a autonomia de seus pacientes, também é seu dever para guiá-los referente dos benefícios e dos riscos de qualquer das suas decisões. Os princípios de bioética do profissional da saúde nos permitem fazer uma avaliação da prática médica, colocando especial atenção para a decisão de escolher de maneira racional uma cirurgia cesariana sobre o parto pela via vaginal.

Palavras-chave: caridade, cesariana, parto, médico, autonomia. 


\section{Introducción}

Objetivamente hablando, existen ciertas necesidades en los individuos que deben ser cubiertas para el correcto y adecuado desarrollo de su persona. Factores como la alimentación, la vivienda y el acceso a los servicios pueden verse como fundamentales, pero sin lugar a duda el tema de la salud se constituye como uno de los elementos determinantes para el desarrollo y consecución del resto de las necesidades (Boltvinik, 1990).

Según el Programa de las Naciones Unidas para el Desarrollo (PNUD), el acceso y la buena calidad en la prestación de servicios de salud puede impactar tanto negativa, como positivamente el futuro de las naciones, a tal grado que tres de los siete objetivos de desarrollo del milenio propuestos para el periodo 2000-2015 se fijaban a partir del acceso y el combate a ciertos problemas de salud, incluidos el combate a enfermedades y la reducción de la mortalidad materna e infantil (PNUD, 2015).

Por causa de esta situación, el lugar que desempeñan los médicos en cuanto a los temas de salud pública ya no solo se enfoca en cómo se cubren las necesidades de sus pacientes, sino también en cómo el ejercicio médico debe apegarse a ciertos requerimientos sociales que permiten estructurar una perspectiva de salud comunitaria, donde los miembros de un Estado consigan desarrollar su vida de manera íntegra y plena.

Lamentablemente, en los últimos años parece ser que el ejercicio profesional de muchos prestadores de servicios de salud se ha visto afectado por el mercantilismo que caracteriza a nuestra sociedad contemporánea, la cual parece tasarse a partir de los bienes de consumo y no del desarrollo de sus integrantes. El comportamiento de todo profesionista, $y$ en especial de aquellos que intervienen con la salud, integridad o los bienes de sus clientes, debe regularse por medio de ciertos parámetros que reflejen lo que se considera como bueno o adecuado para el ejercicio de su profesión, tomando en cuenta su bienestar y el de sus clientes a corto, mediano y largo plazo (Hortal, 2013).

Uno de los temas de salud más sonados en la esfera pública y que ha trascendido a la discusión ética es el referente al uso excesivo y en ocasiones no fundamentado de la operación cesárea, la cual, aunque debería ser una cirugía reservada únicamente a casos en los que el parto por vía vaginal sea riesgoso, en la actualidad se constituye como una alternativa que ya no toma de manera exclusiva y razonada el médico, sino que incluso es una opción que el mismo paciente elige.

El presente artículo busca plantear desde una aproximación ética que la realización de la operación cesárea es una práctica muy controversial en la actualidad y, sobre todo, desde la perspectiva del principio profesional de la beneficencia. Para ello, nos basaremos en los principios de bioética del profesionista de la salud, que no solo nos permiten hacer una valoración del ejercicio de la profesión, 
sino también de los actos que emanan de las decisiones médicas.

De tal manera, se pretende responder a ciertos cuestionamientos centrales: ¿podemos considerar que la práctica injustificada de la operación cesárea viola el principio profesional de beneficencia?, ¿debe el médico poner a consideración del paciente el uso de la operación cesárea como una alternativa de parto? Este artículo busca alcanzar una reflexión acerca de lo óptimo de la práctica de la operación cesárea, considerando aquellas situaciones en las que esta se da como una elección personal de la madre y no así, como un procedimiento quirúrgico necesario que recae exclusivamente en la decisión del médico.

\section{Una aproximación a la operación cesárea}

Objetivamente hablando, el nacimiento es el momento cuando un ser (animal o humano) sale del vientre de su madre, o bien de algún huevo. Sin embargo, aunque en los últimos años el debate político, legal, religioso e incluso ético y filosófico responde a situaciones como la concepción, el nacimiento o los derechos del feto a nacer, el interés del presente artículo no radica en esto, sino más bien en la forma como en las personas este nacimiento (mejor conocido como parto) llega a darse.

El parto es la culminación del periodo de gestación del ser humano, que también viene acompañado por la salida del neo- nato del útero materno. Sin embargo, esta salida no necesariamente tiene que darse de una forma natural o, mejor dicho, por vía vaginal, sino que también existe la posibilidad de que cuando esto no sea posible, el feto sea extraído de la madre a partir de un proceso quirúrgico mediante una operación abdominal llamada cesárea (Organización Mundial de la Salud, 2003).

La primera descripción que se tiene de un parto fue hecha por Hipócrates, en su tratado "Naturaleza del Niño" (Tourtelle, 1828), en el cual planteaba la idea de que el feto nacía comúnmente con la cabeza hacia abajo, debido al peso natural de su cuerpo que era mayor en la mitad superior de este. Posteriormente, según la Suda, el reconocido médico griego Soranus de Éfeso describe todo el proceso con el que debe realizarse el parto, incluyendo el personal necesario, el sitio y los pasos que debían seguirse para facilitar la salida del feto.

En cuanto al tema de la operación cesárea, cabe señalar que este procedimiento ya era practicado desde la antigua Roma e incluso, en ciertos casos especiales, como se contemplaba en la Lex Caesarea, se manejaba con cierta cotidianidad: una mujer que muriese durante el embarazo tardío debía ser sometida a esta intervención con la finalidad de salvar la vida del feto (Jiménez, 1996). Sin embargo, la operación cesárea era en la mayoría de los casos un proceso quirúrgico post mortem, ya que aun realizándolo con la madre viva, la tasa de mortalidad era tan alta que difícilmente sobrevivía al procedimiento (Protho-Medicato, 1795). 
Fue hasta el siglo XVI cuando se tuvo el primer dato de una mujer que sobrevivió a una operación cesárea, lo que modificaba la percepción que tenían los médicos sobre el objetivo final del procedimiento (Cabero y Saldívar, 2009).

En la actualidad, los médicos estiman que la operación cesárea únicamente debería recomendarse para aquellos casos cuando el parto vaginal se presume que puede ser arriesgado para el niño o la madre, como por ejemplo sufrimiento fetal, preeclampsia, nacimientos múltiples, mala colocación del bebe, algún tipo de infecciones, bebes muy grandes, entre otras. Así, la decisión final dependerá en gran medida de la opinión del obstetra responsable, por lo que es inevitable que existan divergencias en cuanto a los puntos de vista de los diferentes médicos sobre la recomendación o no de una operación cesárea (Secretaría de Salud, 2002).

Como lo hemos señalado en un inicio, en las últimas décadas esta situación llega a complicarse aún más, pues la realización de la operación cesárea es puesta a opinión de la madre en muchas ocasiones, es decir, es convertida de un proceso último a un medio alternativo, en el cual la madre puede decidir. Muchos médicos han respaldado la decisión en cuanto a que la madre tiene derecho a decidir como parte de un respeto a su principio de autonomía; sin embargo, ies realmente esta decisión algo que beneficia y no afecta a la futura madre o al neonato?, ¿será objetivamente una elección que se puede tomar de manera racional y óptima por un paciente?, ¿cuál resulta ser el lugar que desempeña el médico en la toma de esta decisión?

Lamentablemente la tasa de nacimientos por medio de una operación cesárea no refleja, en la mayoría de los casos, que esta elección se esté tomando de una forma adecuada, pues considerando que la incidencia normal de cesáreas a nivel mundial debería de ser inferior al $15 \%$ de los nacimientos, es alarmante encontrarnos con algunos países que contemplan una tasa de hasta el 40 $\%$ de los partos; lo cual hace que se convierta en la cirugía más frecuente de sus centros hospitalarios (Vasco y Lopera, 2006). Esto nos lleva a cuestionarnos sobre la realidad con la que se decide el hacer o no una cesárea, y si realmente los principios de responsabilidad y autonomía que mencionan los médicos están justificados sobre los principios de beneficencia y no maleficencia violentados en los pacientes que no requieren necesariamente este tipo de procedimientos quirúrgicos.

\section{La operación cesárea en México}

Con base en el documento de la Secretaría de Salud Cesárea segura: lineamientos técnicos (2002), se entiende por operación cesárea: "La intervención quirúrgica que tiene como objetivo extraer el producto de la concepción y sus anexos ovulares a través de una laparotomía e incisión de la pared uterina" (p. 15). 
De igual manera, este documento nos señala una veintena de causas fundamentales, divididas en tres secciones (causas maternas, causas fetales y causas mixtas), por las que se puede justificar la indicación para la realizar una operación cesárea, esperando que los médicos se apeguen a ellas y de tal forma evitar procedimientos quirúrgicos innecesarios.

Sin embargo, como lo ha señalado el exsecretario de Salud, José Ángel Córdova Villalobos, en México casi el 80 \% de los nacimientos en hospitales privados se llevan a cabo bajo "cesáreas innecesarias", estadística que no supera por mucho a las instituciones públicas, pero que sí resulta alarmante cuando se compara con países desarrollados donde la operación cesárea no sobrepasa el $20 \%$ de los partos (Valadez, 11 de septiembre de 2011).

Lamentablemente, esta situación se ha visto potencializada en los últimos 30 años, en los que la realización de la operación cesárea ha tenido un aumento progresivo a nivel mundial. Solo en México, según la Encuesta Nacional de Salud 2000 y la Encuesta Nacional de Salud y Nutrición (Secretaría de Salud, 2012), el porcentaje total de nacimientos por cesárea reportados durante el periodo 2000-2012 ha aumentado del $30 \%$ al $45 \%$, de los cuales más de la mitad eran procedimientos programados y la mayoría eran innecesarios (Royacelli, 23 de enero de 2013).

Este aumento progresivo y acelerado de partos que se resuelven por medio de una operación cesárea ha alertado, desde hace algún tiempo, a la opinión pública y médica, pues no se considera adecuado que una intervención quirúrgica indicada como alternativa de último recurso termine siendo utilizada $\tan o$ más frecuentemente que el procedimiento original y natural, y sin que esto signifique algún beneficio adicional tanto para la madre como para los niños al nacer (Besio, 1999).

Por ello, es necesario que reflexionemos no únicamente sobre los datos estadísticamente alarmantes del uso y abuso de la operación cesárea en un país como México, sino también que discutamos sobre si las posibles razones que mueven a las futuras madres para elegir una operación cesárea o a los médicos para sugerirlas se encuentran realmente fundamentadas, y si se apegan a lo que debería considerarse como un comportamiento profesional realmente correcto.

Por consiguiente, analicemos el principio ético de beneficencia que los médicos deberían respetar en las decisiones que toman sobre el bienestar de sus pacientes, enfocándonos en los beneficios y riesgos que trae consigo una intervención quirúrgica como lo sería la operación cesárea.

\section{El principio ético de beneficencia}

La beneficencia es un principio que se ha permeado en la ética profesional desde la tradición hipocrática y que hace referencia a la necesidad que tiene todo profesionista de tender, en todo 
momento, a hacer el bien con su cliente. Por consiguiente, el profesional requiere extremar o maximizar los beneficios de sus actos, minimizando todo posible riesgo al que pudiera estar sujeta la persona que contrata sus servicios (Hortal, 2013).

El principio de beneficencia nos hace preguntarnos a qué nos referimos al señalar que se debe hacer el bien o buscar lo bueno en el comportamiento profesional, a lo cual podemos señalar tres aspectos principales (Camacho, 2005):

1. Que el profesionista cumpla con la finalidad de su profesión, equilibrando lo que cobra con la calidad de sus servicios.

2. Que el profesionista promueva una serie de actitudes y conductas que garanticen un clima éticamente positivo entre él y sus clientes, así como con otros miembros del ámbito profesional.

3. Que el profesionista coopere con el bien común, haciendo lo que considera correcto dentro de su sociedad.

Cualquiera de estas tres opciones nos permiten hacernos una idea de la responsabilidad que se concibe al decir que todo profesionista debería buscar en todo momento el bienestar de sus clientes, y evitar así producir daño alguno al sujeto que se está tratando y al mismo tiempo hacer un balance o cálculo prudencial entre los riesgos y los beneficios de sus actos. Para Beauchamp (1979) el principio de beneficencia se sustenta en una obligación moral que tiene la persona de actuar en beneficio de los otros, viendo por ellos en cada una de sus decisiones.

Uno de los mayores problemas que se desencadenan dentro de este principio es el paternalismo que suele suscitarse entre los profesionistas y sus clientes, pues la beneficencia pone una especial atención en el ejercicio de la autoridad que tiene el profesional al ejercer su actividad, lo cual puede confrontarse con la obligación de respetar la autonomía de sus clientes (Busquet, febrero de 2013). Beauchamp (1979) no aceptaba en ningún caso la presencia de actitudes paternalistas dentro del comportamiento profesional, pues consideraba que este tipo de acciones fácilmente degenerarían en un abuso a los clientes.

Apegados a esta postura, otros autores como Diego Gracia y Tristram Engelhardt, consideraban que el principio de beneficencia puede resultar en comportamientos que llegan a ser controversiales, pues es inevitable que se dé cierta tensión entre el deseo del profesionista de buscar el bien de sus clientes y su intención de poner a su consideración las decisiones que se toman en su beneficio.

Para Engelhardt (1995) el principio de autonomía debería ser previamente considerado antes de cualquier actuación del profesionista, pues este se fundamenta en la moralidad del respeto mutuo, con lo cual muestra que el comportamiento profesional debe apegarse al consen- 
timiento previo de sus clientes y así evitar ejercer su autoridad sin valorar los deseos del otro.

Gracia (2008) considera que aunque las tensiones entre el respeto a la autonomía del cliente y el realizar acciones benéficas con base en la autoridad del profesional parecen ser inevitables, es necesario alcanzar un equilibrio entre ambas posturas, pues aunque no podemos aceptar los comportamientos paternalistas característicos de ciertas profesiones, tampoco podemos limitar la actuación profesional por un obligado consentimiento previo.

En el caso específico de las profesión médica, los prestadores de servicio de salud han buscado no verse afectados por esta situación dilemática, previendo para cualquier acción o decisión sobre sus pacientes la firma de un consentimiento informado por parte de los estos. Sin embargo, sigue estando la duda sobre la capacidad de decidir de algunos de los usuarios, pues hacer referencia a la capacidad de autonomía de un individuo nos obliga a prever que este cuente realmente con las características y los elementos cognitivos para ser autónomo.

En la mayoría de los casos, la toma de decisiones de los pacientes se sustenta únicamente en el dicho o información que emana de sus médicos, por lo que la autoridad del profesionista de la salud sigue siendo determinante para la elección, lo que cuestiona la existencia de una verdadera autonomía en el agente decisor. Por ello, aunque la tensión entre el principio de beneficencia y la autonomía parece poder llegar a un punto de acuerdo por medio del consentimiento informado, esto no resulta de todo cierto cuando la autoridad del médico sigue siendo determinante en la decisión de su paciente. Así pues, el médico debe conducirse por el camino de la beneficencia, buscando realmente aquello que para el paciente resulta mejor, independientemente de intereses externos o posibles preferencias poco argumentadas sobre ciertas decisiones médicas.

\section{La búsqueda de la beneficencia de una operación cesárea}

Instrumentalmente hablando, la operación cesárea debe ser un medio quirúrgico que tiende a un fin específico; extraer al producto de la concepción, cuando el parto vaginal podría traer consigo complicaciones médicas (Organización Mundia de las Salud, 2003). Por ello, podemos decir que la operación cesárea debería ser indicada como último recurso y no como un procedimiento alternativo a elección de la madre.

Además, la operación cesárea ha demostrado que no es ni la mejor alternativa de actuación, ni la más segura, pues desde el punto de vista clínico esta puede traer consigo diversas complicaciones, como por ejemplo el debilitamiento del organismo de la madre para próximos partos, la dificultad para alimentar al recién nacido, el aumento de la mortalidad neonatal y materna, así como el aumento del costo económico del parto (Campero et al., marzo-abril de 2007). 
Otra consideración al respecto es la que señalan Alejandro Calvillo, director de la organización civil El Poder del Consumidor, y Katia García, nutrióloga e investigadora alimentaria, quienes plantean que en México el aumento acelerado del número de operaciones cesáreas que se realizan tiene una relación proporcional con la baja alarmante de lactancia materna, pues después del procedimiento quirúrgico es más difícil la formación de leche, pues se retrasa varios días la formación de esta última y ocasiona en muchos casos que el neonato se acostumbre a la formula láctea, siendo este uno de los factores que más comúnmente se relacionan con la obesidad infantil (Proceso, 2013).

Por si esto no fuera poco, no podemos olvidar que la operación cesárea es un procedimiento quirúrgico y que por lo tanto añade riesgos al parto, como por ejemplo la posibilidad de infecciones, la pérdida de sangre e incluso ciertas reacciones o consecuencias no deseadas a causa de la anestesia. También, la cesárea trae consigo un periodo postoperatorio, que en la mayoría de las ocasiones resulta mucho más molesto y prolongado que la recuperación tras un parto vaginal. Sin embargo, a pesar de todos estos factores, las cifras de prácticas de operación cesárea siguen en aumento cada año (Secretaría de Salud, 2002).

Lamentablemente, existen ciertas creencias que han gestado las futuras madres en torno a los beneficios del nacimiento por medio de una operación cesárea, como son aquellas que tienen que ver con la preocupación por el buen nacimiento de sus hijos, o bien las que se dan respondiendo a factores como la incomodidad, el miedo o el dolor que se experimenta durante el parto por vía vaginal. Además, las cosas pueden llegar a complicarse mucho más, cuando la fe del paciente hacia cierto médico lo lleva a seguir la sugerencia de que una operación cesárea que no se necesite es la mejor opción.

Aunque ciertamente es obligación de todo paciente hacerse de toda la evidencia necesaria para alcanzar cierto grado de certeza sobre sus decisiones, no podemos culpar a un individuo que confía en el profesionista que debería ver por el bien de sus intereses y de su persona. Esta situación es la que lleva a que aunque la operación cesárea se practique con el respaldo de un consentimiento informado, buscando respetar la autonomía del paciente, se pueda discutir sobre el comportamiento benéfico que debería promover el profesionista de la salud, pues no podemos asegurar que el actuar de la futura madre es realmente racional, o simplemente una respuesta a la información proporcionada por su médico.

El apego al principio de beneficencia podría modificar claramente la percepción que tienen algunas futuras madres en torno a ciertas situaciones que se dan durante el parto. Por ejemplo, el miedo al dolor del parto por vía vaginal puede ser incoherente en comparación con el dolor de un procedimiento quirúrgico. La comodidad de tener el control de agendar la operación cesárea puede 
ser contraproducente considerando la incomodidad de tener que pasar por un periodo postoperatorio. La postura del médico por realizar un procedimiento innecesario pudiera estar motivada por elementos que no se acercan siquiera a su bienestar, como los beneficios económicos extras que significan la operación cesárea o, incluso, la comodidad misma para el médico.

Es obligación del profesionista de la salud respetar la autonomía y la capacidad de decisión de sus pacientes, pero también es su obligación ver por el bienestar y el beneficio de su actuación profesional. El abuso en el uso de la operación cesárea por motivos innecesarios nos llevan a cuestionar el apego del actuar de los médicos al principio ético de beneficencia al que deberían estar obligados, pues buscar el bien de sus pacientes incluye brindarles toda la información necesaria para tomar una buena decisión y no simplemente quitarse la responsabilidad de su comportamiento a partir de un dudoso consentimiento informado.

Esta situación es otro claro ejemplo en el cual los principios éticos de autonomía y beneficencia se confrontan; sin embargo, el punto central de esta discusión se enfoca en el hecho de que el respeto a la autonomía solo puede configurarse cuando se pueda comprobar que el paciente cuenta con toda la información para tomar una óptima decisión, lo cual en la mayoría de los casos, como lo hemos visto, no es así. Por ende, es necesario que el médico se disponga hacia el paciente como un profesionista que debe apegarse a hacer el mayor bien, lo que incluye dar toda la información sobre las alternativas en el parto, pues solo de esta forma el principio de beneficencia se podría ejercer respetando la autonomía del paciente. Aunque muchos autores argumentan esta tensión entre los principios de bioética, la verdad radica en la elección racional del paciente, la que si no llega a configurarse, difícilmente se puede presumir una verdadera autonomía.

\section{Consideraciones finales}

No cabe duda de que los conocimientos médicos han avanzado de una manera acelerada en los últimos años; no obstante, con todas estas nuevas posibilidades que se abren tanto a los médicos, como a los pacientes, para hacer que sus servicios de salud se apeguen de una mejor manera a sus necesidades, también se trae consigo la obligación de una mayor responsabilidad sobre las elecciones y decisiones que deben tomarse por ambas partes.

Los principios éticos del ejercicio profesional deben poner especial atención en aquellas carreras en las que el cliente suele tener una opinión limitada sobre las actuaciones del profesionista, pues no podemos esperar que un individuo tenga conocimientos profundos de los beneficios, consecuencias y riesgos del actuar profesional; así que deslindar el compromiso al ceder la responsabilidad al cliente no puede ser considerado para todos los casos. 
La práctica excesiva e innecesaria de procedimientos quirúrgicos como la operación cesárea debe combatirse desde el origen mismo de las creencias que respaldan la decisión de la futura madre de preferir este proceso al parto por vía vaginal, pues independientemente de la autonomía del paciente, es obligación del médico actuar siempre en favor y beneficio de su salud y bienestar.

Particularmente en nuestro tema, en principio, la operación cesárea no debería ser una alternativa a disposición de la futura madre sobre cómo llevar a término el parto, pues esto altera completamente el fin mismo del procedimiento. Sin embargo, si la decisión llegase a recaer en la mujer, esta debe hacerse de un conjunto de creencias e información real y verdadera sobre los beneficios y riesgos de esta cirugía, y asegurar de esta manera que su decisión no solo es coherente, lógica y consistente, sino también lo más racionalmente posible, donde el medico busque el mayor bienestar de ella y la de su futuro hijo.

\section{Referencias}

Beauchamp, T. (1979). Principles of Biomedical Ethics. New York: Oxford University Press.

Besio, M. (1999). Cesárea versus parto vaginal. Una perspectiva ética. Revista Médica de Chile, 127(9), 1121-1125.

Boltvinik, J. (1990). Pobreza y necesidades básicas; conceptos y métodos de medición. Caracas: Programa de las Naciones Unidad para el desarrollo [PNUD].
Busquet, E. (febrero, 2013). Principio de autonomía y beneficencia. Dos principios en tensión. recuperado de http://www.bioeticaweb.com/content/view/4576/744/

Cabero, L. y Saldívar, D. (2009). Operatoria obstetrica: visión actual. Buenos Aires: Editorial Médica Panamericana.

Camacho, F. (2005). La ética profesional como pregunta. La lámpara de Diógenes, 81-93.

Campero, L., Hernández, B., Leyva, A., Estrada, F. et al. (marzo-abril, 2007). Tendencias de cesáreas en relación con factores no clínicos en un centro de educación para el parto en la Ciudad de México. Salud Publica, 49(2), 118-125.

Davidson, D. (1995). Ensayos sobre acciones y sucesos. México,D. F: Instituto de Investigaciones Filosóficas de la Universidad Nacional Autónoma de México.

Elster, J. (1983). Uvas amargas. Sobre la subversión de la racionalidad. Barcelona: Peninsula.

Elster, J. (1999). Juicios salomónicos. Barcelona: Gedisa.

Engelhardt, T. (1995). Los fundamentos de la bioética. Barcelona: Paidós.

Gracia, D. (2008). Fundamentos de bioética. Madrid: Triacastela.

Hortal, A. (2013). Ética de las profesiones. Bilbao: Descleé.

Jiménez, R. (1996). Historia de la Cesárea. Maracaibo: Universidad del Zulia.

Organización Mundial de la salud (OMS) (2003). Biblioteca de salud reproductiva. Ginebra: autor.

PNUD. (2015). Programa de Desarrollo de las Naciones Unidas. Recuperado en abril de 2015, de http://hdr.undp.org/ 
en/content/undp\%E2\%80\%99s-multidimensional-poverty-index-2014-specifications

Proceso. (23 de enero de 2013). Nacimientos por cesárea, un negocio redondo en México. recuperado el 4 de abril de 2013, de http://www.proceso.com. $\mathrm{mx} / \mathrm{p}=331456$

Protho-Medicato, R. T. (1795). Modo de hacer la operación cesárea despues de muerta la madre. Buenos Aires: Colegio de Cirugía de San Carlos.

Royacelli, G. (23 de enero de 2013). México, primer lugar en cesáreas. El Universal.

Secretaría de Salud. (2002). Cesárea segura: lineamientos técnicos. Subsecretaría de Prevención y Protección de la Salud,
Dirección General de Salud Reproductiva. México, D. F: autor.

Secretaría de Salud. (2012). Encuesta Nacional de Salud y Nutrición 2012. Resultados nacionales. Secretaría de Salud, Instituto Nacional de Salud Pública. México, D. F: : autor.

Tourtelle, É. (1828). Compendio de la doctrina de Hipócrates. (F. Bonafon, Trans.) Madrid: Imprenta de D. Leon Amarita.

Valadez, B. (7 de septiembre de 2011). 80 por ciento de cesáreas en hospitales privados son innecesarias: SSA. Milenio.

Vasco, M. y Lopera, L. (2006). Técnicas intravenosas para operación cesárea. Revista Colombiana de Anestesiología, 34(1), 35-40. 